\title{
Method for Ankle Foot Orthotics' Mechanical Assessment: A Pilot Study
}

\author{
K. KRUKONIS*, K. DAUNORAVIČIENË $* *$, J. GRIŠKEVIČIUS***, A. KILIKEVIČIUS**** \\ *Vilnius Gediminas Technical University, Basanavičiaus 28a, 03151 Vilnius, Lithuania, \\ E-mail: kestas.krukonis@gmail.com \\ **Vilnius Gediminas Technical University, Basanavičiaus 28a, 03151 Vilnius, Lithuania, \\ E-mail: kristina.daunoraviciene@vgtu.lt \\ ***Vilnius Gediminas Technical University, Basanavičiaus 28a, 03151 Vilnius, Lithuania, \\ E-mail: julius.griskevicius@vgtu.lt \\ ****Vilnius Gediminas Technical University, Basanavičiaus 28a, 03151 Vilnius, Lithuania, \\ E-mail: arturas.kilikevicius@vgtu.lt
}

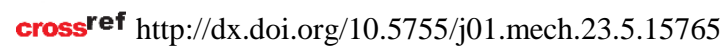

\section{Introduction}

Ankle-foot orthoses (AFOs) are used in clinical practice for a number of indications: from limiting plantar flexion in patients with dropping foot following a stroke to stabilising the mid-stance phase to improving the so-called crouch gait in infantile cerebral palsy, correction of musculoskeletal system and pain reduction. According to international standard ISO 22523, the external orthosis is externally applied device consisting of a single component or an assembly of components applied to the whole or part of the lower limb, upper-limb, trunk, head or neck and their intermediate joints to assist the neuro-muscular and skeletal systems [1]. AFOs can be roughly distinguished by their design and type of material used. In order to assist a person and be comfortable, orthosis fitted individually to each patient. Criteria such as weight, durability and overall aesthetics must be taken into account when choosing an orthosis, moreover, it also must be dynamically and statically stable, have a low shear strength and be biocompatible with the human skin. For this reason, manufacturers often face problems creating the optimal product both functionally and economically. The main question is how much of material should be used for optimal AFO's production that would satisfy all the requirements of the standards, would be reliable, functional and resistant to loads of movement during usage period. AFOs testing and evaluation standards $[1,2]$ regulate features and specifications as well as methodology. However, it should be noted that, in each case of given the re-testing of the product, due to its specificity, and the type of material, additional measures are needed and the conditions that are unregulated. In addition, if the company does not have its certified product-testing laboratory, the product durability tests can be very expensive. Several experimental test models [2] of varying complexity and validity have been described in the literature. However, it is not sufficient to evaluate characteristics of an unused orthosis only, or unworn. The applicable standard ISO 22523 requires the manufacturer to define and inform about required strength and attendant test methods. However, there are no specifications established regarding the test load of the test method $[1,2]$. Therefore, results of clinical research guide towards loading limits and situations supposed to be used into variety of AFOs testing methods and are helpful for collecting initial conditions. In most cases, scientists, studying the orthoses and their applicability and reliability, perform tests of two types: static and dynamic. Static test used to find out the maximum load the orthosis could sustain [3-8]. Depending on the type of AFO and the existing lesions, and other morphological parameters, the force of support for a person standing can range from $180 \mathrm{~N}$ to $900 \mathrm{~N}$. Of course, it depends on the orthosis correct application, the measurement points and way of the usage. It is also important if they were measured on the heel area of the foot or toe [9-11]. AFOs stiffness plays the great importance to orthoses durability and fatigue. As scientists conduct studies using new materials and new technologies, often AFO stiffness is ranging from $0.004 \mathrm{Nm} /{ }^{\circ}$ to $1.8 \mathrm{Nm} /{ }^{\circ}$ or more, depending on the structure of the material and manufacturing [7]. In other works, static tests are carried out in two stages: the first is a creation of a computer model and its analysis using the finite element method (FEM), the second is an experimental testing of the fabricated model. On the other hand, AFO wearers usually are walking and therefore dynamic testing of AFO is important for estimation of dynamic forces and their influence on biomechanics of gait [3, 5, 9-14]. Subjects of specific groups during the tests perform gait with different types of AFOs under appropriate conditions. Such measurements and studies are focused on clinical investigation, AFO efficiency and role in rehabilitation of specific patients. Therefore, compared to static tests, dynamic test collects different temporal and spatial parameters of the gait.

This research work is focused on the task to establish a new and as simple as possible algorithm and/or method for testing AFO's durability. The means of exploring/testing its mechanics were supposed to be as minimal as possible. The testing object - new product (AFO) was obtained from the orthopaedic company with a request to determine weakest points of AFO and strength limits.

\section{Methods and testing protocols}

Based on above made scientific analysis in the literature it was decided to investigate a particular AFO's mechanics according following self-prepared algorithm (Fig. 1, a). Firstly, to perform a finite element analysis (FEA) in SolidWorks and test AFO stress limits, seeking to identify the critical fracture points. Secondly, to perform dynamic experiment on established possible fracture points in differ- 
ent movements' types and conditions; to find forces distributions and maximal values at weakest part of the AFO. Studied object is AFO made of carbon fiber and glass fiber composite, according to a common template $\mathrm{M}$ size. Material characteristics presented in Table 1 .

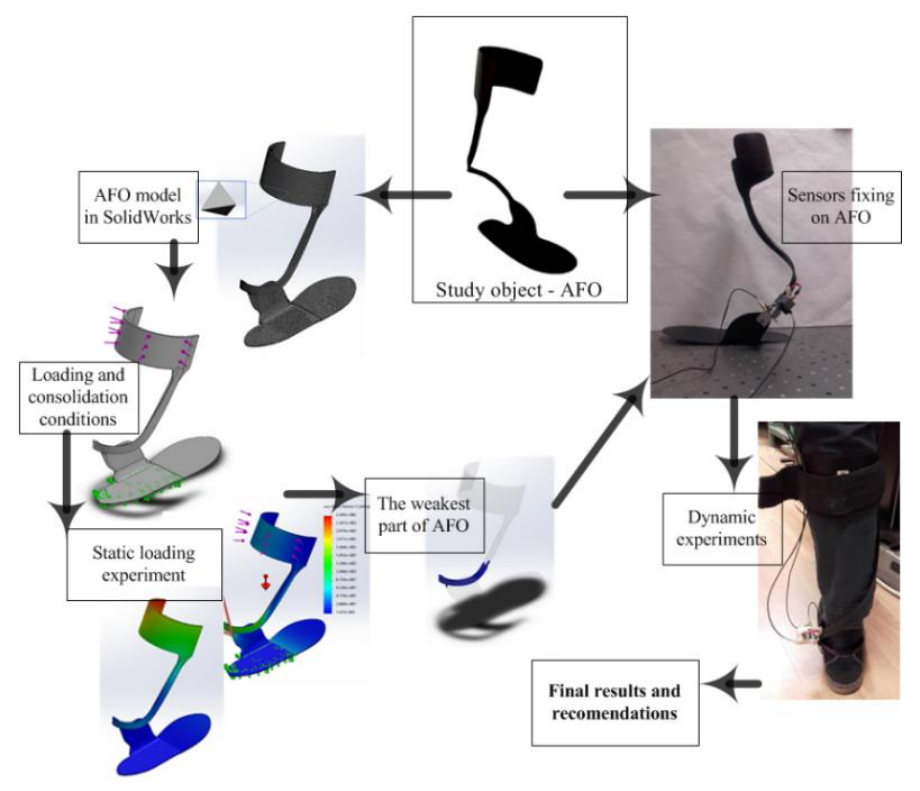

a

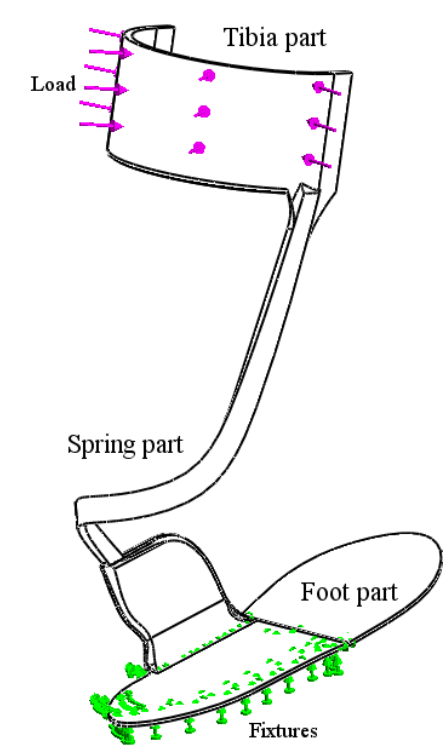

b

Fig. 1 Schemes: a - study procedure, b - AFO model

Mechanical properties of AFO material

\begin{tabular}{|c|c|c|}
\hline Parameter & Value & Dimension \\
\hline The tensile strength & 2735 & $\mathrm{MPa}$ \\
\hline Elastic modulus & 127000 & $\mathrm{MPa}$ \\
\hline Poisson's ratio & 0.20 & - \\
\hline Compressive strength & 1309 & $\mathrm{MPa}$ \\
\hline
\end{tabular}

The model of AFO consists of three parts: the foot, the "spring", the tibia (Fig. 1, b). Each part of AFO dimensions were manually measured and transferred into virtual environment. Computer model was created starting from the foot part by measuring the width of it in every $1 \mathrm{~cm}$. Traces of the fingers as a proportion of the heel part is elevated in every $1.5 \mathrm{~cm}$. "Spring" and the part of the foot connection was simulated by repeating the pattern of real connection. The tibia part has been simulated by repeating actual measured pattern every $2 \mathrm{~cm}$.

Static test has been used with two conditions. Tibia part of AFO was loaded with $150 \mathrm{~kg}$ and the heel portion of the foot was fixed. Following properties of AFO's model were obtained: mass $0.15121 \mathrm{~kg}$; volume $0.0001249 \mathrm{~m}^{3}$; density $1210 \mathrm{~kg} / \mathrm{m}^{3}$.

The model attachment depends on the underlying loads, caused by the user's body weight. The body weight force acts horizontally in the support state. As the foot power during the swing phase does not have a significant impact on the AFO flexibility, this load test is not applicable. Tibia part of a model was loaded with total load of $1500 \mathrm{~N}$ simulating the maximum weight of $150 \mathrm{~kg}$ potential patient/user for AFOs of size M. In other scientific results it was declared that the amount of similar loading should be calculated as subjects' body weight multiplied by coefficient 1.3 [16]. Numerical model of AFO was made assum- ing isotropic and homogenous material properties and modelled as solid body; finite element model of AFO was divided into 42682 tetrahedral shape finite elements. The number of nodes -78228 .

Walking is the main human dynamic movement and it is the most widely studied. Hypothesis was set that it is not necessarily gait could produce maximal forces able to cause dangerous stretch of AFO. Thus, dynamic testing protocol consists of following possible dynamic motions: 1) Gait analysis - performing two full steps. The phase of onestep: support-swing-support. Task repeated 6 times. Gait phases determined from accelerometer data. 2) Squats - carried out on a flat surface landing full body with a splint twice in a row. Task repeated 6 times. 3) Standing vertical jump. Two jumps were made one after another in comfortable way. Task was repeated 3 times. 4) Overcoming the obstacle was expressed as simply stepping on the box and stepping off on the other side at one measure. Task was repeated 3 times. Breaks were organised between all described tasks and between all repetitions as well. Because the investigation is focused on the testing of AFO durability, the number of subjects was not as important as the number of measurements. Two volunteers, weighing 60 and $80 \mathrm{~kg}$, attended the dynamic experiment.

For dynamical study in the AFO's spring part, force transducer and accelerometer were fixed. DeltaTron piezoelectric transducer has a sensitivity of $110 \mathrm{mV} / \mathrm{N}$. Miniature Triaxial DeltaTron Accelerometer with was used for identification of gate cycles. It was glued to the AFO as shown sensors attachments scheme in the Fig. 2. Instrumented AFO was fitted into a shoe of a subject participated in experiment and fixed by stripes in the part of shank (Fig. 1).

Measurements' results were processed and analysed using Excel and Matlab software. Statistical analysis 
was performed to assess differences between different dynamical tasks and one-way ANOVA with significance level $\alpha=0.05$ was used.

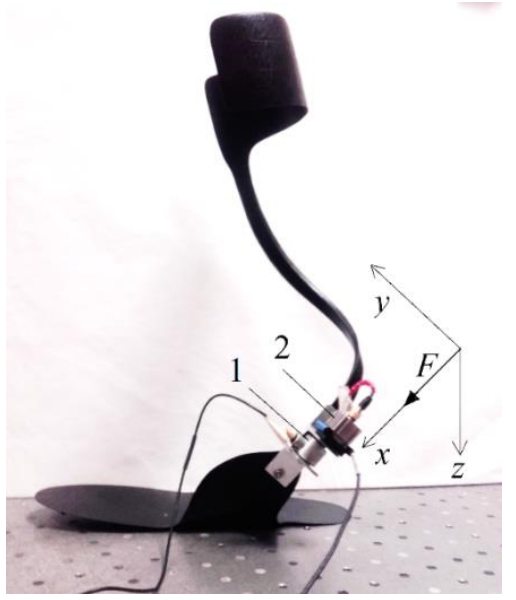

Fig. 2 AFO prepared for dynamical experiment: 1 - force sensor, 2 - accelerometer

\section{Results}

AFO critical breaking point obtained by a static test showed that the largest stress is distributed in the beginning of the spring part. Results showed that the orthosis exposed to $1500 \mathrm{~N}$ load has reached its limit at $1047.8 \mathrm{~N}$, and the maximum stress was $249.5 \mathrm{MPa}$. Thus, the operation under such stresses would create a crack in AFO structure. Static test results are shown in Fig. 3.

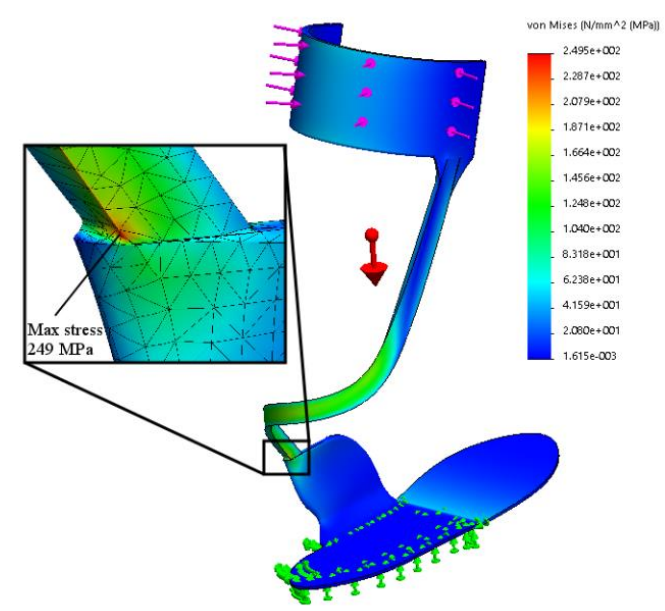

Fig. 3 Maximal stress distribution in AFO' spring part

Table 2

Measurements' data after dynamical experiment

\begin{tabular}{|l|l|c|c|c|}
\hline No & \multirow{2}{*}{$\begin{array}{l}\text { Dynamic mo- } \\
\text { tion }\end{array}$} & \multicolumn{3}{|c|}{ Averaged maximal forces, N } \\
\cline { 3 - 5 } & \multicolumn{3}{|c|}{$F_{W R} \pm \mathrm{SD}$} & $F_{G R} \pm \mathrm{SD}$ \\
\hline 1. & Gait (two & $1^{*}$ & $13.76 \pm 2,19$ & $12.80 \pm 1.70$ \\
\cline { 3 - 5 } & steps) & $2^{*}$ & $15.91 \pm 4.64$ & $18.82 \pm 3.28$ \\
\hline 2. & Squats (two) & 1 & $19.47 \pm 0,85$ & $15.63 \pm 0,98$ \\
\cline { 3 - 5 } & & 2 & $29.52 \pm 4.04$ & $26.78 \pm 5.39$ \\
\hline 3. & Jumps (two) & 1 & $29.01 \pm 2.62$ & $31.68 \pm 6.03$ \\
\cline { 3 - 5 } & & 2 & $29.54 \pm 4.38$ & $33.51 \pm 3.73$ \\
\hline 4. & Overcoming an & 1 & $9.50 \pm 3.86$ & $14.38 \pm 1.34$ \\
\cline { 3 - 5 } & obstacle & 2 & $8.98 \pm 0.44$ & $18.14 \pm 6.45$ \\
\hline
\end{tabular}

* 1 corresponds $60 \mathrm{~kg}$ subject and 2 correspond $80 \mathrm{~kg}$ subject
During dynamic experiment, two types of maximal forces occurring while wearing AFO were measured and analysed. First, subject's weight reaction force $F_{W R}$ was determined in all motion tasks for both participants. Second force is the support reaction force $F_{G R}$, which was measured in the weakest point of AFO as it was estimated by FEA. All measured forces were averaged and standard deviation rates were calculated for each of them (Table 2).

As shown in Figs. 5 and 6 maximum force generated while performing squats and jumps.

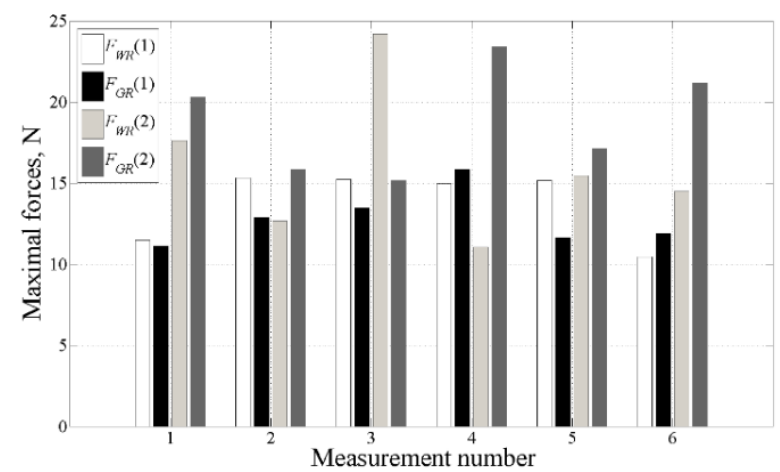

Fig. 4 Forces distribution in gait cycle

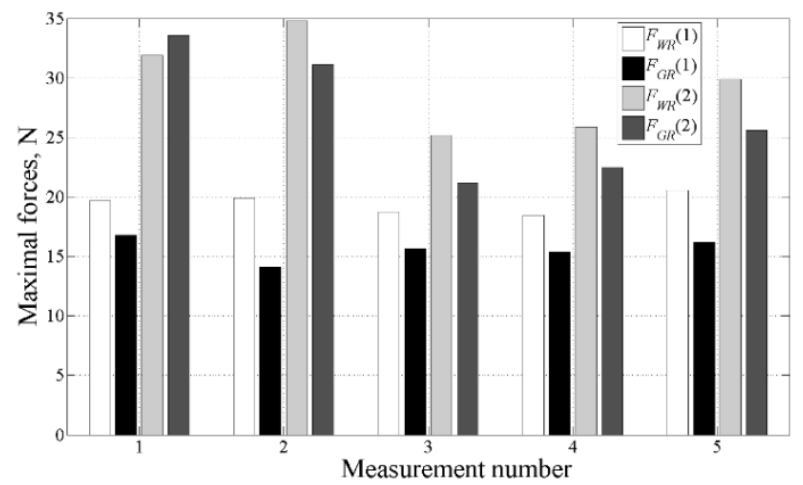

Fig. 5 Forces distribution in squats

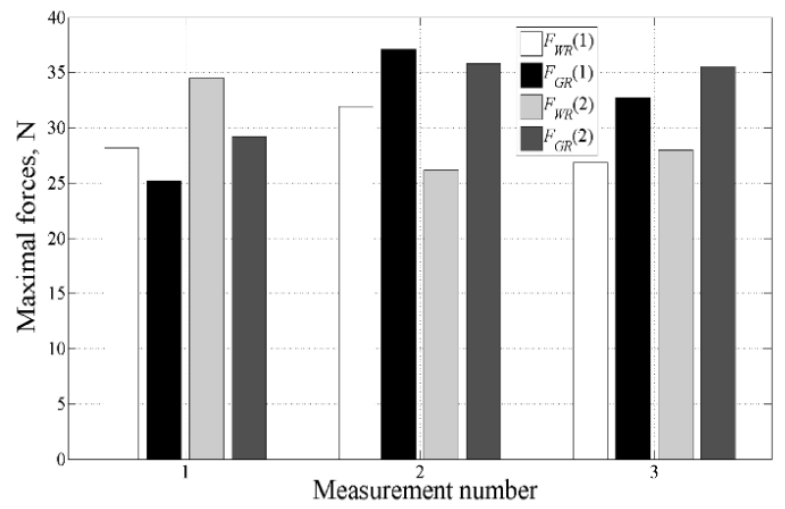

Fig. 6 Forces distribution in jumping

The largest force caused by the body weight and higher amplitude motion affected the AFO mechanics. The major part of the force is determined by the subject weight, but it is already proven and confirmed by the results of other researchers [16]. Higher weight reaction forces $F_{W R}$ observed in the first tests of force measurements and repeating exercises revealed greater support reaction forces $F_{G R}$ in all motion cases (Figs. 4-6). 
Comparison between forces appearing in AFO during different dynamic performance was made and data showed following: statistically significant difference was found between gait and squat $(p=0.01)$, gait and jumps ( $p$ $=0.00)$, gait and obstacle overcoming $(p=0.00)$, jumps and obstacle overcoming $(p=0.01)$.

No other significant differences were found. However, statistical analysis proved that there is no need for selecting special dynamic motion in order to clarify the forces acting on AFO. It could be the same gait and it always suits for analysis on any purpose.

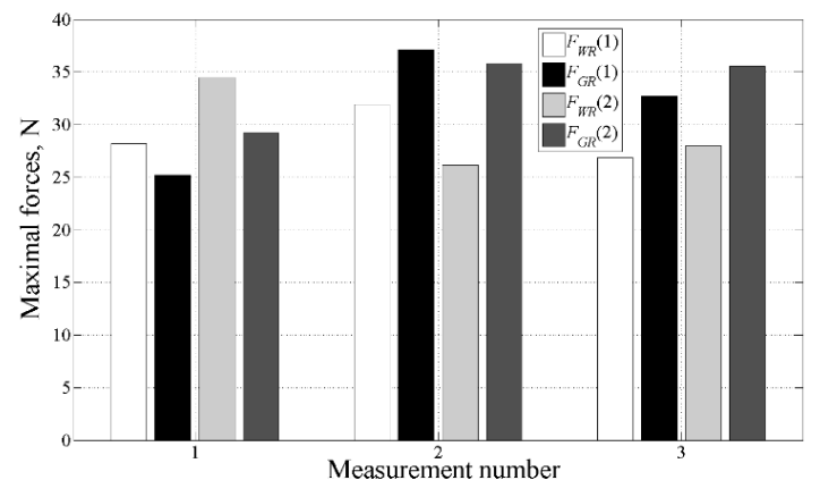

Fig. 7 Forces distribution in climbing the ladder

\section{Discussion}

AFO durability testing method in this work was composed of two stages: static simulation test and dynamic experiments with a real object. AFO model's results have shown that the weakest part of orthosis is the beginning of its spring part. "Spring" prevails in the greatest stresses, therefore, this part is supposed to be increased in area for reducing the stress distribution in it. Other researchers $[4,7$, 15] in their works as well as performing static tests with AFO model and also determined the critical points. Their results were very useful and helpful for comparison with our achievements.

For instance, during the static test using an AFO from carbon fiber was identified the maximum load to which the fracture orthosis follows. At refractive time, the orthosis was loaded with $1970 \mathrm{~N}$ and stress distribution reached to $490.7 \mathrm{MPa}$ [16]. The researchers drew attention to the fact that computer static tests a little different from the experimental. Another scientist Bertram [4] also performed simulation of orthosis, but its main purpose was to determine the force and displacement relation. However, the experiment, noted that the weakest link in orthosis location is also "spring" section and the maximal stresses appear exactly there. Our static test showed AFO critical point and there the force of $1047.8 \mathrm{~N}$ could create a crack in. At this point, the greatest stress was $249.53 \mathrm{MPa}$. The differences between our findings and the literary works have a small difference and we found it normal. It appeared because of different AFO type, material properties, load, and mounting conditions.

Taking into account that it is not sufficient to evaluate characteristics of an unused orthosis only, or unworn, dynamic experiments were performed with a subject wearing AFO in different motion cases. Because we did not seek for clinical results, just for AFO's mechanic testing, we decide that number of people attending the experiment is not so important. However, increasing the number of repetitions of each task we found necessary. Testing object was prepared according recommendations in literature $[1,2]$. Initial conditions and experimental setup was made on the basis of other scientific works' results and clinical also. Gait wearing AFO tests are often in clinical studies [8-13]. It is understood, because the gait is the main human movement ensures its mobility, independence, and because of it, gait phases, cycles, standards and other parameters are so much studied. In their studies scientists are using a special technique, cameras, motion capture, or the like. [8-13]. The research object is a person moving with the AFO and spatialtemporal parameters of gait or in combination with foot pressure distribution are analysing [5, 8-10, 13]. All such works are focused on increasing the efficiency of rehabilitation in clinical field. Since the aim of this study is to examine the durability of the AFO, analysed clinical results were used for creating protocol of experiment, setup main parameters and prediction of possible outcomes. It helped us to avoid critical mistakes in methodology as well as in analysis of results. We assessed that the person wearing AFO is often forced not only simply walk, but also to do more strength, endurance, energy-intensive movements at home or work environment Thus, it was decided to explore the extremessubsequent, expressive movements and their impact on the AFO. Selected dynamic movements were compared with each other in order to find out whether the use of such AFO durability testing methodology is appropriate to choose some kind of purposeful movement and can investigate any motion effects to AFO. Significant difference of maximal forces distribution was found in gait and squat, jumps and obstacle overcoming and jumps and obstacle overcoming. Because no significant difference was found in comparison of gait data with other dynamic motion, it was decided that there is no need for selecting special dynamic motion in order to clarify the forces. Taking into account, that gait is widely analysed in the scientific field, it could be walking and it always suits for analysis on any aims.

Summary of the results from static and dynamic experiments allowed to assess AFO durability and identified its weak point of construction and possible loading conditions. Therefore, methodology could be suitable for simple orthoses' evaluation in manufactory processes and stages or implementation of new product.

\section{Conclusions}

Simulating particular real AFO was valuable to test weakest parts of its structure. It was found that in the spring part of the AFO 1047.8 N loading could cause fracture or crack in its structure. Maximal stresses $249.53 \mathrm{MPa}$ were found at the critical point. Dynamic experiment was helpful to observe real forces distribution at the critical region of AFO in different movements. Distribution of maximal forces was evaluated in all the dynamic conditions of the experiment. The major force acting on the critical point was identified during the jumps $(\sim 30 \mathrm{~N})$. It does not exceed and is far from loading limits estimated in static simulation test. We conclude that our testing results prove suitable orthosis mechanics. Particular AFO's fracture or crack under normal/suitable its wearing conditions is unlikely or even not possible. It can be stated, that AFO produced properly and is safe to use. Our created AFO testing method is sufficient for simple initial assessment. However, it was a pilot study 
and in further our works we are going to improve FEM model by using non homogeneus material and seek for more points of fracture in the particular AFO. Item more detailed strain analysis would be helpful for AFO structure assessment.

\section{References}

1. External limb prostheses and external orthoses - Requirements and test methods. ISO 2006, Switzeland. ISO 22523:2006(E).

2. Hochmann, D. 2014. Testing Procedures for AncleFoot Orthoses. Orthotics. Special edition from Orthopadie Technik 5/14:1-4. Available form Internet: https://verlag-ot.de/con-

tent/e3741823/e3763487/e3763729/e3763737/tiles3763 742/tileEl-

ements3763743/1310_SD_Hochmann_5_14_OB_GB_ ger.pdf.

3. Shorter, K.A.; Kogler, G.F.; Loth, E.; Durfee, W.K.; Hsiao-Wecksler, E. T. 2011. A portable powered anklefoot orthosis for rehabilitation. J Rehabil Res Dev 48(4): $459-472$. http://dx.doi.org/10.1682/jrrd.2010.04.0054.

4. Bregman, D. J. J.; Harlaar, J.; Meskers C. G. M., de Groot V. 2012. Spring-like Ankle Foot Orthoses reduce the energy cost of walking by taking over ankle work. Gait \& Posture 35(1): 148-153. http://dx.doi.org/10.1016/j.gaitpost.2011.08.026.

5. Kim, E. S.; Yoon Y. S.; Sohn M. K.; Kwak S. H.; Choi J. H.; Oh J. S. 2015. Effect of Pneumatic Compressing Powered Orthosis in Stroke Patients: Preliminary Study. Annals of Rehabilitation Medicine 39(2): 226-233. http://dx.doi.org/10.5535/arm.2015.39.2.226.

6. Ramsey, J. A. 2011. Development of a method for fabricating polypropylene non-articulated dorsiflexion assist ankle foot orthoses with predetermined stiffness. Prosthet Orthot Int 35: 54-69. http://dx.doi.org/10.1177/0309364610394477.

7. Zou, D.; He, T.; Dailey, M.; Smith, K. E.;Silva, M. J.; Sinacore, D. R.; Mueller, M. J.; Hastings, M. K. 2014. Experimental and computational analysis of composite ankle-foot orthosis. J Rehabil Res Dev 51(10): 1525-36. http://dx.doi.org/10.1682/JRRD.2014-02-0046.

8. Chen, C. L.; Yeunq, K. T.; Wang, C. H.; Chu, H. T.; Yeh, C. Y. 1999. Anterior ankle-foot orthosis effects on postural stability in hemiplegic patients. Arch Phys Med Rehabil 80: 1587-92. https://doi.org/10.1016/S0003-9993(99)90335-0.

9. Chin, R.; Hsiao-Wecksler, E. T.; Loth, E.; Kogler, G.F.; Manwaring, S. D.; Tyson, S. N.; Shorter, K.A.; Gilmer, J. N. et al. 2009. A pneumatic power harvesting ankle-foot orthosis to prevent foot-drop. Journal of NeuroEngineering and Rehabilitation 6:19. http://dx.doi.org/10.1186/1743-0003-6-19.

10. Ferris, D. P.; Czerniecki, J. M.; Hannaford, B. et al. 2005. An Ankle-Foot Orthosis Powered by Artificial Pneumatic Muscles. Journal of applied biomechanics 21(2): 189-197. http://dx.doi.org/10.1123/jab.21.2.189.

11. Russell, E. E.; Blanck, R. V.; Harper, N. G.; Hsu, J. R.; Wilken, J. M. 2014. How Does Ankle-foot Orthosis
Stiffness Affect Gait in Patients with Lower Limb Salvage? Clinical Orthopaedics and Related Research 472(10): 3026-3035.

http://dx.doi.org/10.1007/s11999-014-3661-3.

12. Telfer, S.; Pallari, J.; Munguia, J.; Dalgarno, K.; McGeough, M.; Woodburn, J. 2012. Embracing additive manufacture: implications for foot and ankle orthosis design. BMC Musculoskeletal Disorders 13:84. http://dx.doi.org/10.1186/1471-2474-13-84.

13. Silver-Thorn, B.; Herrmann, A.; Current, T.; McGuire, J. 2011. Effect of ankle orientation on heel loading and knee stability for post-stroke individuals wearing ankle-foot orthoses. Prosthet Orthot Int 35(2): $150-62$ http://dx.doi.org/10.1177/0309364611399146.

14. Mavroidis, C.; Ranky, R. G.; Sivak, M. L.; Patritti, B. L.; DiPisa, J.; Caddle, A.; Gilhooly, K.; Govoni, L.; Sivak, S.; Lancia, M.; Drillio, R.; Bonato, P. 2011. Patient specific ankle-foot orthoses using rapid prototyping. Journal of NeuroEngineering and Rehabilitation 8: 1 . http://dx.doi.org/10.1186/1743-0003-8-1.

15. Syngellakis, S.; Arnold, M. A.; Rassoulian. 2000. Assessment of the non-linear behaviour of plastic ankle foot orthoses by the finite element method. Proceedings of the Institution of Mechanical Engineers, Part H: Journal of Engineering in Medicine. 214(5): 527. http://dx.doi.org/10.1243/0954411001535561.

16. Zou, D.; He, T.; Dailey, M.; Smith, KE.; Silva, MJ.; Sinacore, DR.; Mueller, MJ.; Hastings, MK. 2014. Experimental and computational analysis of composite ankle-foot orthosis. J Rehabil Res Dev 51(10): 1525-36. http://dx.doi.org/10.1682/JRRD.2014-02-0046.

K. Krukonis, K. Daunoravičienė, J. Griškevičius, A. Kilikevičius

\section{METHOD FOR ANKLE FOOT ORTHOTICS' MECHANICAL ASSESSMENT: A PILOT STUDY}

S u m m a r y

This paper presents the task to establish a new and as simple as possible algorithm and method for testing ankle-foot orthoses (AFO) durability. Finite element analysis of the AFO allowed estimate weak points in the structure of orthosis. It was found that in the spring part of the AFO 1047.8 N loading could cause fracture or crack in its structure. Maximal stresses $249.53 \mathrm{MPa}$ were found at the critical point. Distribution of maximal forces was evaluated in all the dynamic conditions of the experiment. The major force acting on the critical point was identified during the jumps $(\sim 30 \mathrm{~N})$. It does not exceed and is far from loading limits estimated in static simulation test. Created AFO testing method is sufficient for simple initial assessment.

Keywords: ankle-foot orthoses, durability, FEA, accelerometer, force measurement.

Received July 20, 2016

Accepted October 13, 2017 